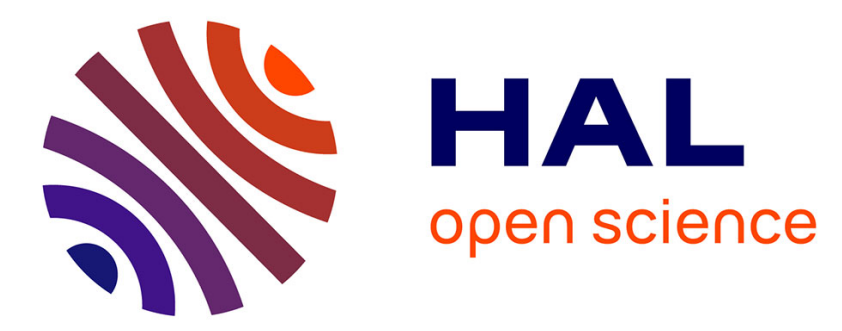

\title{
Des effets paradoxaux de l'action publique patrimoniale: entre valorisation des spécificités urbaines et standardisation des espaces centraux historiques
}

\author{
Mathieu Gigot
}

\section{- To cite this version:}

Mathieu Gigot. Des effets paradoxaux de l'action publique patrimoniale: entre valorisation des spécificités urbaines et standardisation des espaces centraux historiques. Norois, 2018, Patrimoine, vignoble, continuité écologique, transition énergétique, anthropologie des catastrophes, 249, pp.7-20. 10.4000/norois.7090 . hal-02101240v2

\section{HAL Id: hal-02101240 \\ https://hal.science/hal-02101240v2}

Submitted on 1 Jul 2020

HAL is a multi-disciplinary open access archive for the deposit and dissemination of scientific research documents, whether they are published or not. The documents may come from teaching and research institutions in France or abroad, or from public or private research centers.
L'archive ouverte pluridisciplinaire HAL, est destinée au dépôt et à la diffusion de documents scientifiques de niveau recherche, publiés ou non, émanant des établissements d'enseignement et de recherche français ou étrangers, des laboratoires publics ou privés. 


\section{DES EFFETS PARADOXAUX DE L'ACTION PUBLIQUE PATRIMONIALE ENTRE VALORISATION DES SPÉCIFICITÉS URBAINES ET STANDARDISATION DES ESPACES CENTRAUX HISTORIQUES}

\section{Mathieu GIGOT}

Chercheur associé au centre Jean-Bodin, université d'Angers et à l'UMR CITERES, CNRS, université de Tours

\section{Résumé :}

Cet article analyse l'action publique patrimoniale dans trois villes du Val de Loire (Angers, Tours et Orléans). Les mécanismes d'interventions patrimoniales qu'il met en évidence, aboutissent-ils à produire une image standardisée des espaces centraux patrimonialisés? En décomposant les outils mis en œuvre dans chacune des trois villes, l'article identifie leurs effets paradoxaux ainsi que les spécificités et les modes de faire de chacune des villes. Il montre que des espaces types (dé)marquent aujourd'hui les centres-villes patrimonialisés, dans un contexte territorial concurrentiel. En même temps, ces interventions urbaines ont aussi pour effet de diffuser des modalités de marquage des espaces publics, des modèles de mobilier urbain et de traitement des façades qui produisent ou renforcent l'identification d'un type d'espace urbain spécifique. La généralisation de modes d'intervention, de stratégies d'image et de marquage de l'espace instaurent un nouveau type d'espace : le centre historique. L'action patrimoniale sur les centres anciens agrège une série de caractéristiques qui en font des espaces standardisés, reconnaissables aux signes produits par leur patrimonialisation.

Mots clefs : Patrimoine, Centre historique, Action publique, Angers, Tours, Orléans

\section{Abstract : \\ Cities of the Loire Valley : Paradoxical Effects of Public Heritage Action}

This article analyses local public action concerning cultural heritage among three cities in the Loire Valley (Angers, Tours, and Orleans). It seeks to respond to the question if appropriated cultural heritage intervention mechanisms produce a standardised image of central cultural heritage spaces. By deconstructing the tools already implemented by each of the three cities, this article identifies their contradictory effects as well as the methodical specificities of each historical city. It is shown that model spaces set apart cultural city-centres in a competitive territorial context. As a secondary effect, these urban interventions also disseminate methods for marking public spaces, as well as urban furniture and building façade treatment, which in turn, produces and reinforces the identification of a singular and specific urban space. The homogenisation of these intervention methods as well as of urban representation and demarcation strategies, establishes a new type of space: the historical city-centre. The cultural heritage measures taken in historic city-centres aggregates a multitude of characteristics which creates standardised spaces, recognizable by the produced effects caused by their heritagisation.

Keywords: Cultural heritage, Historic city center, Public action, Loire Valley 


\section{INTRODUCTION}

La patrimonialisation des espaces urbains est un processus qui s'est généralisé en France depuis les années $1960^{1}$ concernant en premier lieu les quartiers anciens des villes. Fortement dégradés, ces quartiers étaient devenus les symboles de la crise des centres, mais restaient partie prenante des espaces centraux fortement chargés de valeurs symboliques. Jean-Paul Lévy rappelle en effet que le centre « est l'espace le plus marqué sur le plan symbolique [...] Plus que tout autre espace, il est émetteur de signes et de symboles » (Lévy, 1987). Dans ce cadre, l'action publique patrimoniale a pris une importance considérable dans des contextes urbains qui pour beaucoup étaient taxés d'insalubres et menacés de destruction. L'organisation du sauvetage des centres anciens, promis à une rénovation brutale, a pu être analysée comme une forme de réaction à la globalisation des sociétés et des formes urbaines dans la mesure où préserver les quartiers anciens s'apparente à une sauvegarde de l'identité locale. En effet, si la construction patrimoniale est fortement liée au renforcement de l'État central et à l'idée d'un patrimoine de la nation ${ }^{2}$, Guy Di Méo, a bien montré en quoi le patrimoine pouvait aussi être un catalyseur de l'identité locale : pour lui, « toute société localisée recherche des attaches et des valeurs territoriales. Elle s'efforce toujours d'ancrer son rapport spatial dans la longue durée, réelle ou mythifiée » (Di Méo, 1996). Appréhendé comme une construction sociale (Veschambre, 2007), le patrimoine contribue à façonner une histoire collective propre à affirmer des spécificités locales.

L'appel au passé ferait donc figure de résistance à l'uniformisation du monde de sorte que les sociétés contemporaines tendent à conserver leur patrimoine comme des pans d'une histoire qui leur échappe, absorbée par le tourbillon de l'urbanisation et de la globalisation. C'est là un paradoxe frappant de cette patrimonialisation généralisée des centres anciens car «le patrimoine a ainsi perdu sa fonction constructive au profit d'une fonction défensive qui assure la recollection d'une identité menacée » (Choay, 1996). Face à de nouveaux projets urbains, les nombreuses mobilisations des associations locales montrent bien comment l'argument patrimonial est invoqué : il est menacé, mis en péril, etc. et doit être protégé ${ }^{3}$. C'est d'ailleurs contre une forme de standardisation de la ville que ces associations luttent activement en souhaitant conserver les héritages du passé. Le patrimoine est, de ce point de vue, un marqueur identitaire et sa conservation «s'impose alors comme la voie royale de la protection du

\footnotetext{
${ }^{1}$ Notamment avec l'apparition des secteurs sauvegardés créés pour protéger et valoriser le patrimoine urbain (loi $\mathrm{n}^{\circ}$ 62-903 du 4 août 1962, dite loi Malraux).

${ }^{2}$ Héritage de la Révolution française, l'idée de patrimoine en tant que bien commun de la nation s'est traduite en particulier par la loi du 31 décembre 1913 qui inventa les monuments historiques qu'elle dote d'une protection.

${ }^{3}$ À cet égard, on peut noter la création depuis le début du XX ${ }^{\mathrm{e}}$ siècle de grandes associations patrimoniales, aujourd'hui réunies dans un « G8 du patrimoine ».
} 
symbolique » (Jeudy, 2001), c'est-à-dire de ce qui fait l'unicité d'une ville. Les villes semblent avoir besoin des immeubles anciens pour se développer, pas seulement là où la valeur patrimoniale est la plus forte (Jacobs, 1991).

Cet article montrera que dans trois villes du Val de Loire (Angers, Tours, Orléans), la valorisation des spécificités urbaines contribue en parallèle à standardiser les centres anciens en créant une nouvelle catégorie d'espace directement identifiable, et ce quelle que soit la ville : le centre historique patrimonialisé ${ }^{4}$.

Les trois terrains choisis sont tous concernés par des zonages patrimoniaux qui, même s'ils ne sont pas de même nature juridique, montrent une forme de reconnaissance du patrimoine urbain par les institutions, locales et/ou nationales. Les centres-villes de Tours et d'Orléans sont aussi compris dans le périmètre du Val de Loire inscrit au patrimoine mondial de l'humanité au titre des paysages culturels. Ces trois villes font partie de la même aire géoculturelle et partagent un substrat culturel commun, qui se matérialise notamment au travers de l'architecture et des paysages : utilisation récurrente de matériaux (tuffeau et ardoise), répétition de formes paysagères... Ce sont d'ailleurs ces éléments qui fondent les critères de la valeur universelle exceptionnelle et l'intérêt patrimonial du Val de Loire (Coyaud, 2003).

Il n'est donc pas question de montrer que ces trois contextes se ressemblent mais davantage de comprendre en quoi l'action publique patrimoniale locale, qui s'est mise en place dans des temporalités différentes dans les trois contextes, a généralisé des modes d'intervention et produit des espaces types répondant à des critères standardisés.

\section{DES STRATÉgIES DE DÉMARCATION DANS UN CONTEXTE TERRITORIAL CONCURRENTIEL}

La patrimonialisation des espaces urbains s'inscrit dans un contexte fortement concurrentiel entre les collectivités locales. Le patrimoine est progressivement devenu, depuis les années 1980, un atout pour répondre aux attentes des touristes et des habitants en quête d'un cadre de vie agréable.

Les interventions patrimoniales, c'est-à-dire toutes les formes de mise en valeur des espaces reconnus comme ayant une valeur historique - comme par exemple l'attention portée aux

\footnotetext{
${ }^{4}$ Cet article est le fruit d'une recherche menée dans le cadre d'une thèse de doctorat entre 2007 et 2012 . Des entretiens complémentaires ont néanmoins été conduits en 2018 à l'occasion d'un programme de recherche ANR sur le Plan Local d'Urbanisme patrimonial (CJB, université d'Angers / CITERES, CNRS, université de Tours).
} 
façades, au traitement des espaces publics ou au mobilier urbain ${ }^{5}$ - se sont généralisées depuis les années 1980 dans les centres-villes. Ces modalités d'interventions tendent à produire un modèle urbain implicite, fondé sur des catégories d'usages, d'images et de pratiques. Il est flagrant de constater que les acteurs locaux mettent en place les mêmes stratégies sur le traitement et la qualification de leurs centres anciens - notamment dans l'objectif d'améliorer l'attractivité touristique - tout en produisant un discours qui met l'accent sur les spécificités locales de ces espaces. Antonio Paolo Russo a montré en quoi le développement du tourisme dans les centres historiques contribue à renforcer des espaces qui ne concentrent que certains types d'activités et d'attractions. Il évoque d'ailleurs un « "cercle vicieux" du développement touristique » (Paolo Ruso, 2002). Les trois villes que nous étudions ont, à un moment ou à un autre, connu ce processus de mise en tourisme avec la promotion d'espaces types dont les traitements ont été assez similaires. Certes, cela s'inscrit dans une dynamique plus large puisque la requalification des centres-villes est un phénomène qui a pris de l'ampleur à partir des années 1980. Après la « crise des centres », qui a fait l'objet de nombreuses recherches en géographie et sociologie urbaine des années 1960 aux années 1980 (Lévy, 1987 ; Soucy, 1980) et qui est le produit de facteurs conjugués comme l'ampleur des destructions de la seconde guerre mondiale, les mutations socio-économiques des années 1950 ou le délabrement progressif de l'habitat, on observe dans le courant des années 1980 une mutation de ces espaces, «caractérisée par une amorce de recentrage de la dynamique urbaine qui, après s'être manifestée dans les franges urbaines et périurbaines depuis les années 1960, touche beaucoup plus intensément les quartiers anciens » (Madore, 1994).

Avec la crise des centres, marquée par une profonde détérioration de l'habitat, des espaces publics et des conditions de vie, les élus locaux ont tenté de reconquérir les quartiers anciens dégradés et d'en faire un signal fort, un marqueur territorial leur permettant de se démarquer, en valorisant ce qui fonde la spécificité de leur ville car «à l'inverse de son abandon dans les années 1960, le centre-ville doit [maintenant] être beau et valoriser l'image de la ville » (Dumont, 2010). Pour ce faire, les élus locaux font appel aux «éléments qui composent la symbolique d'une ville [et qui] sont de nature hétérogène. Ils relèvent à la fois des monuments, de la morphologie urbaine... » (Tiano, 2005). Le patrimoine est alors souvent mobilisé dans les stratégies de marketing urbain car il serait justement l'artefact de l'authenticité des lieux. Dans un article sur le patrimoine de marque, Fabien Pécot et Virginie De Barnier, deux chercheurs en management, montrent comment l'appel au passé des villes permet de construire une image

\footnotetext{
${ }^{5}$ Ces modes d'intervention font appel à des outils de droit commun qui ne dépendent pas de législations reprises dans le Code du patrimoine.
} 
de marque spécifique (Pécot et De Barnier, 2015) : les communicants mobilisent l'image de la ville historique pour développer l'attractivité du territoire. L'objectif n'est pas uniquement d'attirer des touristes mais aussi de nouveaux habitants et de nouvelles entreprises. Le centre ancien véhicule l'image de la ville où il fait bon vivre, dotée d'une ambiance urbaine spécifique au point de devenir un espace archétypal repris dans les stratégies de marketing urbain. Or, Fabien Pécot et Virginie De Barnier remarquent que « les managers en charge des marques de ville font l'objet de critiques car les stratégies dominantes tendent à faire se ressembler les villes entre elles dans le discours et la réalité » (Pécot et Barnier, 2015). En particulier, ces stratégies de communication font massivement appel au patrimoine comme élément du cadre de vie et facteur de différenciation.

L'image des centres anciens patrimonialisés se focalise donc sur leurs spécificités qui tiennent à la fois à l'architecture, la morphologie urbaine et l'ambiance du lieu. Un certain nombre de travaux - en particulier chez les urbanistes - se sont attachés à saisir ces ambiances urbaines qui participent à définir des catégories de lieux. Les interventions urbaines en centre ancien, dans toute leur diversité, contribuent à construire ces ambiances et les images de la ville patrimonialisée même si pour Alain Bourdin, « l'ambiance l'emporte sur le lieu, la connotation sur la dénotation, l'effet psychologique sur le trait de caractère » (Bourdin, 1984). Les stratégies des acteurs publics s'orientent en effet davantage vers la création d'espaces types, dotés d'une certaine ambiance de nature à en faire des espaces de convivialité.

Comme l'explique Maria Gravari-Barbas en évoquant l'esthétique de la ville touristique, « la mise en valeur du patrimoine est pour beaucoup dans la "théâtralisation" de la ville » (GravariBarbas, 1998). Ce ne serait donc pas la protection du patrimoine qui uniformise les villes, mais les choix de mise en valeur.

\section{TEMPORALITÉS DU PATRIMOINE ET DYNAMIQUES URBAINES DANS TROIS VILLES DU VAL DE LOIRE}

Les centres-villes sont les lieux symboliques qui représentent aujourd'hui l'essence même de la ville et sur lesquels se basent les stratégies de communication territoriale. Plus encore, «ils constituent la mémoire de la ville, par la présence de monuments et de bâtiments historiques, le tracé et le nom des rues » (Clavel, 2002). Rien d'étonnant - donc - à ce que les centres-villes aient été préservés, de façon à ce que la ville se donne à lire à travers eux. C'est d'ailleurs le sens de la mise en récit de la ville par les édiles locaux. 


\section{A. Patrimonialisation et nouveaux usages du centre ancien à Tours}

À Tours, ville moyenne ligérienne, Jean Royer, maire de 1959 à 1995, met en œuvre le plan de rénovation du centre-ville mais ressent «ces propositions apparemment logiques par leur modernisation comme une offense au passé car ces quartiers furent ceux d'une ville prospère » (Royer, 1997). Il choisit alors d'utiliser les outils de la rénovation urbaine pour engager une restauration du centre ancien, via la définition d'un périmètre opérationnel de 9 hectares. Avec cette expérimentation, le maire de Tours cherche à retrouver la grandeur (exagérée sans doute) du centre ancien comme en atteste le titre éloquent de son ouvrage : «La cité retrouvée » (Royer, 1977).

En valorisant son patrimoine, la Municipalité tourangelle a mis en place les conditions d'un retour au centre. Lorsque l'on analyse les temporalités de l'action patrimoniale à Tours, on voit bien que la protection du patrimoine a tout de suite été liée à sa mise en valeur. Dès 1961, la Municipalité met en place un secteur de restauration avant même que la loi sur les secteurs sauvegardés ne soit votée en 1962. La restauration des immeubles et la requalification des espaces publics ont construit une nouvelle centralité et de nouveaux usages récréatifs qui n'existaient pas auparavant dans ce quartier de la ville. Le centre ancien de Tours - du moins pour sa partie occidentale - a muté du quartier résidentiel populaire au quartier récréatif par excellence, comme en témoignent la concentration de débits de boissons et de restaurants : en 2018, on ne dénombre pas moins de 88 Licence IV dans le vieux Tours ${ }^{6}$.

La création du secteur sauvegardé au début des années 1970 a été l'occasion d'étudier la ville ancienne en profondeur afin de créer un outil qui permet d'encadrer et de règlementer son évolution. Si des campagnes massives de restauration du bâti ancien avaient déjà été entamées depuis les années 1960, le secteur sauvegardé a ouvert des avantages fiscaux aux propriétaires désirant restaurer leurs immeubles ${ }^{7}$. En parallèle, la Municipalité a mené des campagnes de ravalements de façades, obligatoires et subventionnées afin d'embellir le centre ancien. Plusieurs Opérations Programmées d'Amélioration de l'Habitat (OPAH) se sont aussi succédées dans la décennie 1980. Puis, dans la deuxième moitié des années 1980, la Municipalité entreprend la piétonisation du quartier et la requalification de ses espaces publics (Figure 1). Le processus de patrimonialisation prend une ampleur considérable et le quartier devient la figure de proue de la ville, à tel point que la place centrale du quartier (place Plumereau) en vient à symboliser la ville patrimoniale. Michel Lussault note d'ailleurs que le

\footnotetext{
${ }^{6}$ Source : service Commerce de la Ville de Tours (2018).

${ }^{7}$ La loi Malraux permet de défiscaliser des impôts sur le revenu le montant de travaux de restauration, sous conditions.
} 
quartier historique de Tours, celui qui constitue le symbole même de la vieille ville, « est en général considéré comme un beau décor par nombre de ceux qui la pratiquent, et non comme un territoire marqué par le patrimoine monumental et historique au sens strict du terme, d'aucuns, plus critiques, y voyant même un simulacre, un pastiche de quartier "médiéval" » (Lussault, 1993). «Décor»: le mot est lâché ; ce type de vocabulaire revient souvent dans le discours des acteurs locaux, comme ce membre de l'équipe municipale qui qualifiait le secteur sauvegardé de Tours de «vitrine de la ville ${ }^{8}$. Il faut dire que la transformation du quartier depuis les années 1960 et les premières opérations de restauration immobilière qui se sont multipliées à la fin des années $1970^{9}$ ont entrainé la mutation des usages, passant d'un quartier résidentiel à une vaste zone de loisirs, encore habitée mais sujette à de nombreux conflits liés au bruit notamment. Patrice Melé (2004) avait ainsi identifié, à partir d'entretiens auprès de résidents du quartier Plumereau, de nombreuses représentations d'un quartier « disqualifié comme bruyant, dangereux et sur la voie d'une tendance inéluctable à la dégradation ». Néanmoins, ce «vieux Tours » incarne ce qui fait d'une ville une ville, c'est-à-dire une forme d'urbanité lisible à travers la qualité du paysage, l'ambiance urbaine, une vie commerciale, un lieu de sociabilité certaine qui voit se croiser dans un même espace plusieurs types de populations, le quartier étant en effet autant fréquenté par les Tourangeaux que par les touristes de tous horizons. Michel Lussault (1993) a bien montré que ce quartier incarne depuis longtemps (avant même son réinvestissement) «l'image de Tours ». Il évoque une «image patrimoniale » ancienne qui légitime les nombreuses interventions publiques dans ce quartier. Cette évolution est d'autant plus marquante que le quartier de la Cité, autour de la cathédrale Saint-Gatien plus à l'Est, lui aussi protégé et lui aussi soumis à des périmètres d'interventions patrimoniales, n'a pas connu le même sort. Si les interventions patrimoniales, sur l'espace public notamment, ont été les mêmes, le quartier de la Cité est par contre resté résidentiel. Le modèle du centre historique n'est donc pas uniquement lié aux politiques publiques et aux mécanismes d'interventions patrimoniales : il se construit aussi selon des dynamiques sociospatiales particulières. La spécialisation commerciale, notamment, produit des espaces types : à Tours comme dans la grande majorité des grandes villes et des villes moyennes françaises, on retrouve une typologie d'espaces commerciaux particuliers : axes commerciaux où les franchises dominent, rues où la proportion de bars et restaurants est importante, etc. Ces formes de spécialisations contribuent à l'homogénéisation des images urbaines. Les réhabilitations de

\footnotetext{
${ }^{8}$ Entretien avec un élu de la ville de Tours, 2009.

${ }^{9}$ Après la mise en place du secteur sauvegardé tourangeau en 1973 et grâce aux possibilités de défiscalisation Malraux, de nombreuses opérations de restauration immobilière d'initiative privée se sont enchaîné.
} 
l'habitat et la valorisation des quartiers anciens conduisent aussi à des phénomènes d'embourgeoisement observés à Tours, en particulier dans le quartier de la Cité contrairement aux abords de la place Plumereau.

\section{B. La théâtralisation du centre-ville orléanais : une question d'image ?}

Le même type de processus a été observé à Orléans, mais avec un décalage temporel notable. Jusqu'au début des années 2000, il n'y avait pas de politique patrimoniale d'envergure à Orléans, ce qui se traduisait notamment par l'absence de document de gestion spécifique. Les archives de courriers échangés entre le maire et le conservateur des monuments historiques dans les années 1960 attestent de la naissance avortée d'un secteur sauvegardé ${ }^{10}$, qui n'a jamais vu le jour à cause des opérations de rénovation urbaine trop avancées dans certaines parties du centre historique, alors dégradé et lentement déserté (Vannier, 2001). Il semble que cet échec ait été vécu comme un traumatisme par les Municipalités successives qui ne se sont jamais saisies de l'opportunité de mettre en place un outil d'urbanisme patrimonial, même si le maire Roger Secrétain a tenté, au milieu des années 1970, d'intégrer des prescriptions de sauvegarde dans son Plan d'Occupation des Sols ${ }^{11}$. Il faudra attendre l'élection d'une nouvelle équipe municipale menée par Serge Grouard en 2001 pour que le centre historique revienne au cœur du projet politique ${ }^{12}$ et constitue le «"cœur de ville", objet spatial fondamental» (Dumont, 2005). Les élus décideront de travailler prioritairement à la requalification des espaces publics du centre-ville. Le goudron sera alors remplacé par des pavés en pierres de Souppes et la généralisation de ce type de revêtement, tout comme la suppression généralisée des trottoirs dans le cadre de la piétonnisation progressive du centre-ville ${ }^{13}$, donneront une certaine unité aux rues de la ville pourtant construites à des époques différentes.

Dans le même temps, une campagne de ravalements de façades obligatoire et subventionnée sera mise en œuvre sur le périmètre du centre historique et la Municipalité, en lien avec les services de l'État, s'engagera dans l'étude d'une Zone de Protection du Patrimoine Architectural, Urbain et Paysager (ZPPAUP) afin d'encadrer les autorisations d'urbanisme sur l'ensemble du centre historique et quelques faubourgs. De l'aveu même des différents acteurs

\footnotetext{
${ }^{10}$ Délibération du Conseil municipal d'Orléans abandonnant le principe d'un secteur sauvegardé, 31 mai 1963.

${ }^{11}$ Rapport du directeur général des services techniques sur l'approbation du projet d'aménagement pour la mise en valeur des quartiers anciens, présenté par Guy Nicot, architecte en chef. Archives municipales d'Orléans, cote 8214.

${ }^{12}$ Une dizaine de pages est consacrée au centre-ville dans le projet urbain baptisé «Orléans 2015, promenonsnous demain », édité en 2005.

${ }^{13} 21$ rues du centre ancien sont rendues aux piétons en 2002.
} 
locaux ${ }^{14}$, ce sont les premières interventions, en particulier les ravalements de façades, qui ont fait prendre conscience aux élus de l'importance du patrimoine à Orléans. Jusque-là cachés sous des couches de crépis, les pans de bois ont été dévoilés dans le centre ancien depuis le lancement de la campagne de restauration des façades en $2002^{15}$. Pour les élus et l'architecte des bâtiments de France, le centre ancien constitue alors un «laboratoire à ciel ouvert » où chacun (re)découvre le patrimoine ${ }^{16}$. Si la Municipalité engage une politique patrimoniale sur son centre historique, c'est dans l'objectif de le mettre en valeur et d'initier une réappropriation de l'espace, tant par les habitants que par les touristes. En effet, Orléans est aux portes des châteaux de la Loire, au sein du site inscrit UNESCO, mais la ville peine à trouver son public et souffre d'une mauvaise image, d'un trouble identitaire, que Michel Grésillon (1995) attribue à « une symbiose difficile à établir entre patrimonial et moderne ». La revalorisation du centre ancien, qui découle des différentes interventions patrimoniales de protection ou de mise en valeur, doit permettre de capter les touristes du Val de Loire et d'impliquer les habitants dans le façonnement d'une meilleure image de la ville. Dans cette optique, la Municipalité entreprend, moins d'un an après la mise en place de la ZPPAUP et quelques années seulement après les premiers ravalements de façades, de demander le label Ville d'art et d'histoire, qu'elle obtient finalement en 2009 (Figure 1).

On peut évoquer ici une certaine mise en scène du centre historique orléanais car les interventions sur le patrimoine n'ont touché que les façades du centre historique sans que ne soient pensées de façon systématique les réhabilitations de logements. Ce façadisme se retrouve dans les mots utilisés dans le magazine municipal par exemple qui, dans son numéro de mai 2018, produit un article consacré aux façades rénovées et explique que «le visage de la ville apparaît métamorphosé depuis le lancement du plan de restauration $\gg^{17}$. De visage, il est aussi question si l'on évoque l'omniprésence de la promotion de la figure de Jeanne d'Arc dans la ville : au-delà des fêtes johanniques annuelles, la municipalité a créé un emblème représentant la pucelle d'Orléans et son étendard. Le même type de phénomène a pu être observé plus récemment à Tours avec la célébration de l'anniversaire de la naissance de Saint-Martin, figure locale redécouverte et magnifiée par la nouvelle Municipalité en place qui, pour l'occasion, a créé un logotype et des fêtes spécifiques. Ces récits autour de grands personnages de l'histoire

\footnotetext{
${ }^{14}$ Entretiens avec l'architecte des bâtiments de France du Loiret, l'élu à l'urbanisme de la Ville d'Orléans et plusieurs techniciens des services Urbanisme et Patrimoine de la ville d'Orléans, 2010.

${ }_{15} 1.000$ façades ont été restaurées en 16 ans.

${ }^{16}$ Entretien avec le Chargé de mission Restauration du patrimoine, service du Patrimoine de la ville d'Orléans, 2010.

${ }^{17}$ « Façades rénovées : en plein dans le mille !», Orléans.mag, n¹58, mai 2018, p. 16-17.
} 
emportent avec eux un certain nombre de marqueurs urbains (clous à l'effigie des personnages plantés dans le sol, pochoirs graffés sur les trottoirs, parcours thématiques, omniprésence des figures historiques dans la ville et ses documents de promotion) que l'on retrouve de façon récurrente dans les centres anciens. Ils participent, en plus des autres interventions en centre ancien, à construire une narration patrimoniale, c'est-à-dire « la construction d'une relation entre les visiteurs et le monde du passé et leurs habitants »(Flon, 2012), comme l'a expliqué Émilie Flon à propos des vestiges archéologiques.

Il faut donc distinguer à Orléans trois processus concomitants. D'une part, la Municipalité entreprend bien une patrimonialisation du centre-ville au travers de la mise en place d'un document de protection du patrimoine, d'autre part, elle engage une politique de réhabilitation des façades des édifices (mais pas forcément des intérieurs) et enfin la promotion touristique fait rayonner l'évocation de Jeanne d'Arc à partir de signes dans l'espace urbain. Ce faisant, les acteurs locaux glissent clairement vers une politique de façadisme, qui n'est pas à proprement parlé patrimoniale, car il s'agit avant tout de « privilégier l'ordonnancement urbain, l'image de la ville, au détriment de la structure et de l'usage des bâtiments » (Loyer et Schmuckle-Mollard, 2001), donc de créer un décor de théâtre. Par la mise en scène (et en lumière notamment) de son patrimoine, la Municipalité a voulu magnifier son centre ancien de façon à en révéler ses caractéristiques. Si l'outil réglementaire de protection du patrimoine est différent à Orléans, les mécanismes d'intervention sur le centre ancien sont les mêmes qu'à Tours, à quelques nuances près : dans les restaurations de pans de bois, la Municipalité d'Orléans a pris le parti de restituer les couleurs du pan de bois à un instant donné ${ }^{18}$, ce qui pose d'ailleurs la question de l'authenticité du paysage urbain ainsi produit qui devient le pastiche d'époques différentes choisies pour chaque façade. En dehors de cette spécificité, le traitement du centre historique répond globalement aux caractéristiques récurrentes de la réhabilitation: le quartier est piétonnisé, l'espace public est réorganisé de façon à accueillir des terrasses de cafés, etc.

\section{Angers : interventions patrimoniales sans protections dédiées}

À Angers, un site patrimonial remarquable est actuellement à l'étude sur le centre-ville, voire au-delà, mais jusqu'alors, la protection du patrimoine n'avait pas bénéficié d'un outil d'urbanisme patrimonial ad hoc, ce qui n'a pas empêché les Municipalités successives de mettre en place de nombreuses interventions dans le centre ancien : piétonisation du centre-ville en

\footnotetext{
${ }^{18}$ Grâce à la technique de dendrochronologie qui permet de connaître les différentes teintes de bois au fil du temps.
} 
1976, campagnes de ravalements de façades à partir des années 1980 et quatre OPAH (Figure 1). Comme à Tours et Orléans, les mécanismes d'intervention se sont tournés vers la valorisation de l'image et du cadre de vie, produisant un type d'espace marqué par des signes et chargé de valeurs spécifiques propres à différencier le centre historique du reste de la ville. Très tôt, la ville a fait un effort considérable dans l'identification du patrimoine urbain en ouvrant en 1978 un service municipal de l'inventaire. Cette initiative et les différentes interventions patrimoniales entreprises, notamment autour des grands monuments historiques (le château, la cathédrale et la maison d'Adam) ont permis à Angers d'obtenir le label Ville d'art et d'histoire en 1986 même sans document de gestion du patrimoine, ce qui reste assez rare. En effet, 89\% des communes labellisées Ville ou Pays d'Art et d'Histoire disposent d'un outil d'urbanisme patrimonial qui assure la protection du patrimoine. Le cas angevin montre que l'intervention patrimoniale peut s'inscrire dans l'espace urbain sans outil de protection du patrimoine territorialisé, c'est-à-dire sans zonage spécifique qui impliquerait une codification symbolique et juridique des espaces. En effet, en l'absence d'un zonage patrimonial à Angers, ce sont les jeux d'acteurs qui ont dicté la doctrine en matière de protection du patrimoine dans le cadre des autorisations d'urbanisme négociées entre la Municipalité et l'architecte des bâtiments de France aux abords des monuments historiques : «ce qui n'était pas écrit était largement partagé dans une politique » selon l'architecte des bâtiments de France en poste dans les années $2000^{19}$. Cependant, en l'absence de document de gestion, les différentes Municipalités se sont laissées une certaine forme de latitude quant aux rénovations à entreprendre dans le centre-ville. Vincent Veschambre a bien montré comment la mise en valeur ou la destruction du patrimoine angevin ont été des marqueurs de l'action publique locale dans la mesure où « démolition et conservation constituent les deux faces d'un même processus de réinterprétation et de revalorisation permanente des espaces urbains » (Veschambre, 2005). Les associations locale ${ }^{20}$ ont tenu un rôle majeur dans la protection du patrimoine angevin. Si le centre ancien de la ville a été valorisé au travers des interventions précitées, la pérennité de la préservation du patrimoine n'était pas clairement assurée, ce qui a conduit ces associations à se positionner en gardiens du patrimoine urbain non protégé. Pour l'administratrice de la Fédération Sauvegarde de l'Anjou, « la Ville affiche une politique patrimoniale qui n'est pas la sienne $»^{21}$ puisqu'elle se retranche derrière l'État qui gère ses monuments historiques et leurs abords. Néanmoins, force est de constater que l'absence d'outils ad hoc ne change pas la nature

\footnotetext{
${ }^{19}$ Entretien avec l'architecte des bâtiments de France du Maine-et-Loire, 2009.

${ }^{20}$ Parmi lesquelles l'association Renaissance de la Doutre ou l'association Sauvegarde de l'Anjou.

${ }^{21}$ Entretien avec une administratrice de l'association Sauvegarde de l'Anjou, 2009.
} 
des interventions en centre ancien : on retrouve ici à la fois la protection contre les destructions et interventions abusives menée par la ville et l'architecte des bâtiments de France dans le cadre abords des monuments historiques et des politiques d'image et de marquage des rues du centre, même si les opérations de rénovation ont été plus importantes à Angers que dans les deux autres cas. Par ailleurs, si la proportion de commerces de bouche est moins importante qu'à Tours ou Orléans, le même système de terrasses de café qui s'étendent sur les rues piétonnisées a pu être observé dans certaines parties du centre ancien.

\section{L'INVENTION D'UN MODÈLE DU « CENTRE HISTORIQUE »}

L'analyse fine des trois terrains montre comment, dans des temporalités différentes, la patrimonialisation des espaces urbains a produit des centres anciens semblables. De ce point de vue, la patrimonialisation des centres anciens participerait, paradoxalement, à remodeler des espaces de façon à ce qu'ils soient facilement identifiables, avec des signes uniformisés qui permettent de les reconnaître et de marquer l'existence de l'acte de patrimonialisation : une sorte « d'esperanto touristique » pour reprendre l'expression de Maria Gravari-Barbas (1998). C'est comme si, en substance, étaient créées les conditions qui permettent de dire que « le centre historique, c'est ici ». D'ailleurs, la multiplication dans le monde des sites inscrits au patrimoine de l'UNESCO pose bien la question d'une uniformisation de traitement du patrimoine puisque des recommandations concernent le paysage urbain historique ${ }^{22}$. Cédric Feriel évoque « la mise en identité de la ville » pour décrire le développement d'un modèle de traitement des centresvilles européens, en particulier autour des politiques de piétonnisation (Feriel, 2015). Ce processus s'opère d'ailleurs de façon concomitante dans plusieurs villes d'Europe où les politiques urbaines en centre-ville prennent des formes similaires (Ashworth et Tunbridge, 1990). En somme, « les espaces produits du patrimoine urbain ne sont pas les survivances d'une histoire ancienne ou des lieux de résistance face à l'uniformisation amenée par la modernité capitaliste. Ils sont les produits mêmes de cette modernité » (Bonard et Felli, 2008). La patrimonialisation généralisée des espaces urbains a donc participé à créer un nouveau modèle urbain : le « centre historique patrimonialisé ».

En France, la loi LCAP de $2016^{23}$ a confirmé la volonté du législateur de rendre plus lisibles les espaces patrimonialisés, en harmonisant les dispositifs de protection au travers de

\footnotetext{
${ }^{22}$ Résolution $n^{\circ} 41$ « Recommandation concernant le paysage urbain historique », Programme et budget pour 20122013, Unesco.

${ }^{23}$ Loi no2016-925 du 7 juillet 2016 relative à la liberté de la création, à l'architecture et au patrimoine.
} 
l'appellation unique de « site patrimonial remarquable ». En outre, l'exposé des motifs rappelle que « les patrimoines sont un immense atout de la France qu'il convient de promouvoir et de valoriser afin de répondre aux attentes des publics et des territoires ». C'est donc une logique de valorisation des patrimoines qui est poursuivie à travers le texte de loi, de sorte que les espaces patrimonialisés correspondent à un certain standard patrimonial.

Nous avons vu comment les interventions semblables sur les trois terrains d'étude tendent à produire un type d'espace, c'est-à-dire un produit patrimonial identifiable facilement. Paradoxalement, les supports de communication décrivent ces espaces patrimoniaux comme des hauts lieux de la ville, ceux qui en fondent l'âme. Les trois villes se sont d'ailleurs engagées, à un moment ou à un autre, dans la démarche d'obtention du label Ville d'art et d'histoire. Les labellisations dans le domaine du patrimoine participent assez largement à l'uniformisation du «produit patrimonial » car « la multiplication des labels et des espaces protégés entraine une standardisation du patrimoine, en répétant inlassablement un modèle commun de mise en valeur répondant au même cahier des charges »(Gigot, 2016). Les labels développent des modèles d'interventions patrimoniales, lissés pour répondre aux attentes des usagers, habitants ou touristes. Villes et pays d'art et d'histoire, petites cités de caractère, Plus beaux villages ou Plus beaux détours de France : autant de labellisations pour lesquelles les différents cahiers des charges proposent une lecture relativement unifiée du patrimoine.

Ainsi, dans la charte de qualité du label Plus beaux villages de France comme dans celle des Petites cités de caractère, l'un des critères d'appréciation des candidatures réside dans la qualité du patrimoine, notion qui recouvre notamment la question des matériaux, du traitement des espaces publics, de l'éclairage, du mobilier urbain, etc. L'enjeu est louable puisqu'il s'agit avant tout de ne pas dénaturer le site mais le label vise tout autant à produire les conditions de l'identification des villages labellisés qui, d'ailleurs, affichent cette labellisation sous les panneaux d'entrée de ville comme pour signifier l'entrée dans un lieu particulier, reconnu pour sa valeur patrimoniale. Cet affichage ostentatoire du patrimoine permet au touriste d'identifier facilement le type d'espace dans lequel il entre et il sait, au final, ce qu'il trouvera dans les villes et villages labellisés. Autrement dit, les narrations patrimoniales locales, constructions intangibles, produisent des espaces tangibles standardisés car l'édification de récits suit le même processus fondé sur la mise en valeur des patrimoines locaux.

Ainsi, sur l'ensemble du réseau des villes et pays d'art et d'histoire, les mêmes formats de visites sont développés ${ }^{24}$, seul le contenu change, lié à l'histoire urbaine locale. Le terme de

\footnotetext{
${ }^{24}$ Comme par exemple la collection « Laissez-vous conter... » développée dans les villes d'art et d'histoire.
} 
réseau est d'ailleurs lourd de sens puisque plusieurs associations de collectivités promeuvent la protection du patrimoine et tentent d'essaimer les bonnes pratiques au travers de l'organisation de journées d'études ou de publications thématiques. C'est le cas par exemple de l'association Sites et cités remarquables de France qui, forte d'environ 200 collectivités adhérentes, s'efforce de véhiculer des modèles de protection, de valorisation du patrimoine, mais aussi d'intervention sur l'image urbaine des espaces centraux ce qui participe d'une certaine façon à la standardisation des centres anciens.

\section{CONCLUSION}

La généralisation des interventions urbaines dans les centres-villes patrimonialisés participe à la production d'un standard urbain : restauration des façades, piétonisation, traitement des espaces publics, notamment par le mobilier urbain, sont autant d'éléments qui permettent de repérer ce qui constitue le centre historique d'une ville. La Figure 2 montre de façon schématique comment le centre ancien patrimonialisé agrège des outils similaires dans les trois villes étudiées et en quoi il se distingue des espaces qui l'entourent. Pour autant, ces interventions urbaines en centre-ville ne sont pas systématiquement reliées au patrimoine : c'est en particulier le cas pour les politiques de piétonnisation, davantage justifiées par le renforcement de l'attractivité commerciale de centres-villes en déclin (Feriel, 2015). La patrimonialisation des centres anciens et leur mise en valeur produisent donc des effets paradoxaux : l'attention des collectivités dans les trois centres-villes étudiés se justifie par la volonté de mettre en exergue les spécificités urbaines et pourtant les interventions urbaines se font selon des codes standardisés. La patrimonialisation des espaces urbains et la généralisation de ces interventions urbaines créent donc une nouvelle catégorie d'espace: le «centre historique », en tant qu'objet spatial qui répond à une représentation standardisée des caractéristiques qu'il doit recouvrir. Ces qualifications découlent des interventions de sauvegarde et de valorisation du patrimoine qui confèrent à l'espace une série de valeurs qui le rendent identifiable et conforme à ce que la société en attend. Ainsi, le centre historique est chargé symboliquement et correspond à un certain modèle de ville.

Nous avons montré comment dans trois villes du Val de Loire, ces interventions se sont systématisées avec des temporalités différentes (Figure 1). Du point de vue de l'image urbaine et du marquage d'un espace patrimonialisé, les résultats sont aujourd'hui similaires. Les modalités et temporalités d'intervention sont différentes mais leurs effets ont été les mêmes, 
tant et si bien que l'on retrouve dans ces trois villes un vocabulaire commun dans le traitement du centre historique, avec de légères différences notamment liées au vieillissement inégal des aménagements.

Par ailleurs, il n'est pas anodin de rappeler que ce phénomène n'est pas propre à la ville ancienne puisque certaines opérations immobilières contemporaines tendent à reproduire les modèles de la ville ancienne, en pastichant l'architecture locale ${ }^{25}$. Les promoteurs immobiliers proposent des opérations consensuelles qui reprennent - voire recopient - le vocabulaire architectural du centre historique au détriment du geste architectural contemporain : c'est particulièrement vrai dans les projets de nouveaux centres commerciaux. En périphérie de Tours, un promoteur envisage de construire « un véritable village avec ses rues, ses places pittoresques qui rappelleraient celles des cités historiques de Touraine et du Val de Loire $»^{26}$, voulant ainsi reproduire ex nihilo le modèle du centre historique que nous décrivions. Cela s'inscrit dans une idéologie aujourd'hui dominante où l'on « ne prétend plus imposer aux citadins des formes interchangeables et épurées, dénuées de toute référence à l'esprit des lieux » (André, 1994). L'analyse des documents de planification urbaine (pas uniquement patrimoniaux) est à cet égard frappante car tous prônent la bonne insertion dans l'environnement existant, mais contraignent, de ce fait, un renouvellement urbain novateur dans ses formes.

Les interventions patrimoniales qui visent la mise en valeur du patrimoine continuent de produire des catégories d'espaces identifiables facilement dont les caractéristiques tendent à être lissées, même si chaque ville conserve ses spécificités architecturales et urbaines qui la différencient toujours des autres. La patrimonialisation des centres anciens et le traitement dont ils font l'objet institutionnalisent le patrimoine urbain qui devient une catégorie cognitive. Il semble important qu'il soit bien là où on l'attend, avec les caractéristiques que l'on en attend, permettant une expérience urbaine reproductible en différents lieux : la promenade du touriste confronté à des références historiques difficilement assimilables mais marquée par une ambiance rassurante et valorisée. Les usages de ces espaces patrimonialisés s'inscrivent aussi dans cette dynamique d'uniformisation des centres historiques, avec la récurrence de certains types de commerces et de pratiques (bars et restaurants, artisans...) l'embourgeoisement des quartiers et le développement du tourisme et des usages récréatifs.

\footnotetext{
${ }^{25}$ Cela peut être, à certains égards, à rapprocher du rôle des architectes des bâtiments de France que nous ne pouvons développer dans notre propos.

${ }_{26}$ « Bientôt un village des marques à Sorigny ? », La Nouvelle République du 2 juin 2018.
} 
Les modalités de l'action publique patrimoniale dans les centres anciens et les dynamiques spatiales qui en découlent posent la question d'une «nouvelle crise des centres historiques » (Melé, 2004) causée par les nuisances liées la spatialisation des fonctions touristiques, de restauration et de divertissement dans un espace. De plus, la banalisation de l'image des centres historiques et des usages qui s'y développent risque de les déposséder de leurs spécificités urbaines.

\section{Bibliographie}

André J.L., 1994. Au coeur des villes, Odile Jacob, Paris, 186 p.

Ashworth G.J., Tunbridge J.E., 1990. The tourist - Historic city, Belhaven Press, London, 265 p.

Bonard Y., Felli R., 2008. Patrimoine et tourisme urbain. La valorisation de l'authenticité à Lyon et Pékin, Articulo - Journal of Urban Research, n 4, p. 1-14. DOI : 10.4000/articulo.719 Bourdin A., 1984. Le patrimoine réinventé, Presses Universitaires de France, Paris, 239 p.

Choay F., 1996. L'allégorie du patrimoine, Éditions du Seuil, Paris, 270 p.

Choay F., 2009. Le patrimoine en questions : anthologie pour un combat, Éditions du Seuil, Paris, $215 \mathrm{p}$.

Clavel M., 2002. Sociologie de l'urbain, Antrhopos, Paris, 123 p.

Coyaud L.-M., 2003. Paysages culturels du Val de Loire, 303, arts, recherches et créations, $\mathrm{n}^{\circ} 75$, p. $7-11$

Di Méo G., 1996. Production des identités et attachement au lieu, in Lamy Y. (dir.), L'alchimie du patrimoine : discours et pratiques, p. 247-271.

Dumont G.F., 2010. Déclin ou renouveau des centres-villes ?, in Dumont G.F. (dir.), La France en villes, p. 269-276

Dumont M., 2005. Le développement urbain dans les villes intermédiaires: pratiques métropolitaines ou nouveau modèle spécifique? Le cas d'Orléans et Tours, Annales de Géographie, ${ }^{\circ} 642$, p. 141-162. DOI : 10.3917/ag.642.0141

Feriel C., 2015. L'invention du centre-ville européen. La politique des secteurs piétonniers en Europe occidentale, 1960-1980, Histoire urbaine, n42, p. 99-122. DOI : 10.3917/rhu.042.0099 Flon E., 2012. Les mises en scène du patrimoine: savoir, fiction et médiation, Lavoisier, Cachan, $223 \mathrm{p}$. 
Gigot M., 2016. Labels et outils d'urbanisme patrimonial : quelle articulation ?, Juris art etc., $\mathrm{n}^{\circ} 41$, p. $27-30$.

Gravari-Barbas M., 1998. Belle, propre, festive et sécurisante : l'esthétique de la ville touristique, Norois, ${ }^{\circ} 178$, p. 175-193.

Grésillon M., 1995. Orléans, Anthropos, Paris, 160 p.

Hugues F., Hirczak M., Sénil N., 2006. Territoire et patrimoine : la co-construction d'une dynamique et de ses ressources, Revue d'économie régionale et urbaine, n ${ }^{\circ}$, p. 683-700. DOI : $10.3917 /$ reru.065.0683

Jacobs, J., 1991, Déclin et survie des grandes villes américaines, Pierre Mardaga Éditeur, Liège, $435 \mathrm{p}$.

Jeudy H.P., 2001. La machinerie patrimoniale, Sens\&tonka, Paris, 127 p.

Lévy J.P., 1987. Centres-villes en mutation. Éditions du CNRS, Paris, 257 p.

Lévy J.P., 1987. Réflexions sur l'évolution contemporaine des centres-villes, Bulletin de l'association des géographes français, $\mathrm{n}^{\circ} 4$, p. 307-315.

Loyer F., Schmuckle-Mollard, C., 2001. Façadisme et identité urbaine, Éditions du patrimoine, Paris, $382 \mathrm{p}$.

Lussault M., 1993. L'assomption d'une ville, in Fresnault-Deruelle P. (dir.), Dans la ville, l'affiche, Maison des Sciences de la Ville, Tours, p. 98-107.

Lussault M., 1993. Tours : image de la ville et politique urbaine, Maison des Sciences de la Ville, Tours, $415 \mathrm{p}$.

Madore F., 1994. Marchés immobiliers des espaces centraux et dynamiques sociales de moyennes villes françaises, in Chevalier J. et Peyon J.-P. (dir.), Au centre des villes : dynamiques et recompositions, p. 15-44.

Melé, P., 2004. Habitants mobilisés et devenir d'un espace patrimonial ?, Géocarrefour, $\mathrm{n}^{\circ}$ 3, vol. 79, p. 223-230. DOI : 10.4000/geocarrefour.734

Paolo Russo A., 2002. The "vicious circle" of tourism development in heritage cities, Annals of tourism research, vol. $29, \mathrm{n}^{\circ} 1, \mathrm{p} .165-182$

Pécot F., De Barnier V., 2015. Stratégies de marques de ville basées sur le patrimoine de marque : le rôle des symboles, Management et avenir, ${ }^{\circ} 78$, p. 143-159.

Royer J., 1977. La cité retrouvée, Presses de la Cité, Paris, 123 p.

Royer J., 1997. Il était une fois...un maire, CLD, Chambray-lès-Tours, 173 p.

Soucy C., 1980. La crise des centres : orientation de la recherche, contribution à une sociologie des centres urbains, Centre de sociologie urbaine, Paris, 164 p. 
Tiano C., 2005. La dimension symbolique de la ville: une réponse à la concurrence métropolitaine ?, Lieux communs, ${ }^{\circ} 8$, p. 75-102.

Vannier S., 2001. Orléans au fil de son histoire, CLD, Chambray-lès-Tours, 127 p.

Veschambre V., 2007. Patrimoine : un objet révélateur des évolutions de la géographie et de sa place dans les sciences sociales. Annales de Géographie, ${ }^{\circ} 656$, p. 361-381. DOI: 10.3917/ag.656.0361

Veschambre V., 2005. Le recyclage urbain, entre démolition et patrimonialisation : enjeux d'appropriation symbolique de l'espace. Norois, $\mathrm{n}^{\circ} 195$, p. 79-92. DOI : 10.4000/norois.548

$* * * * *$ 

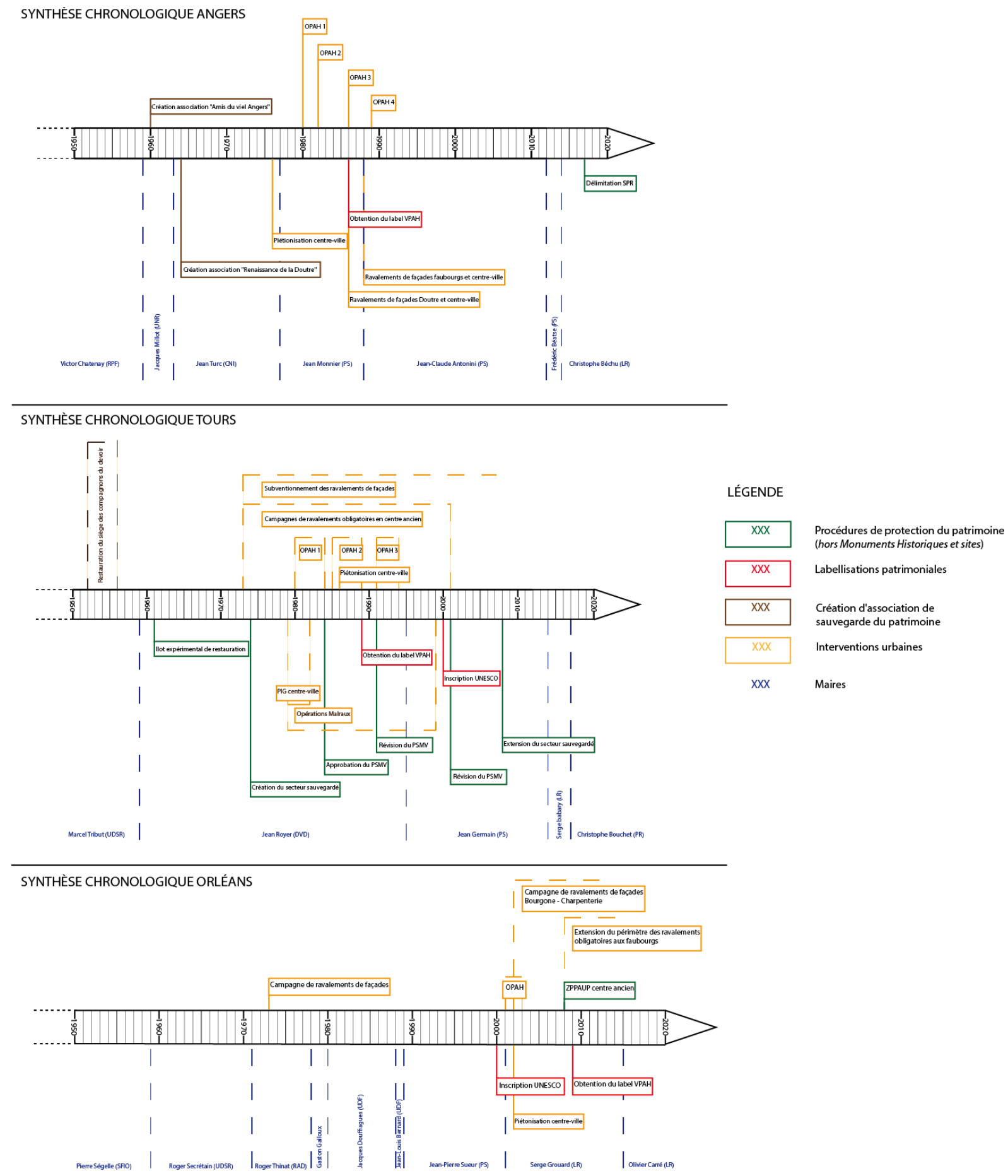

Figure 1 : Frise chronologique synthétique de l'action publique patrimoniale à Angers, Tours et Orléans / Synthetic timelines of cultural heritage public action in Angers, Tours and Orléans 

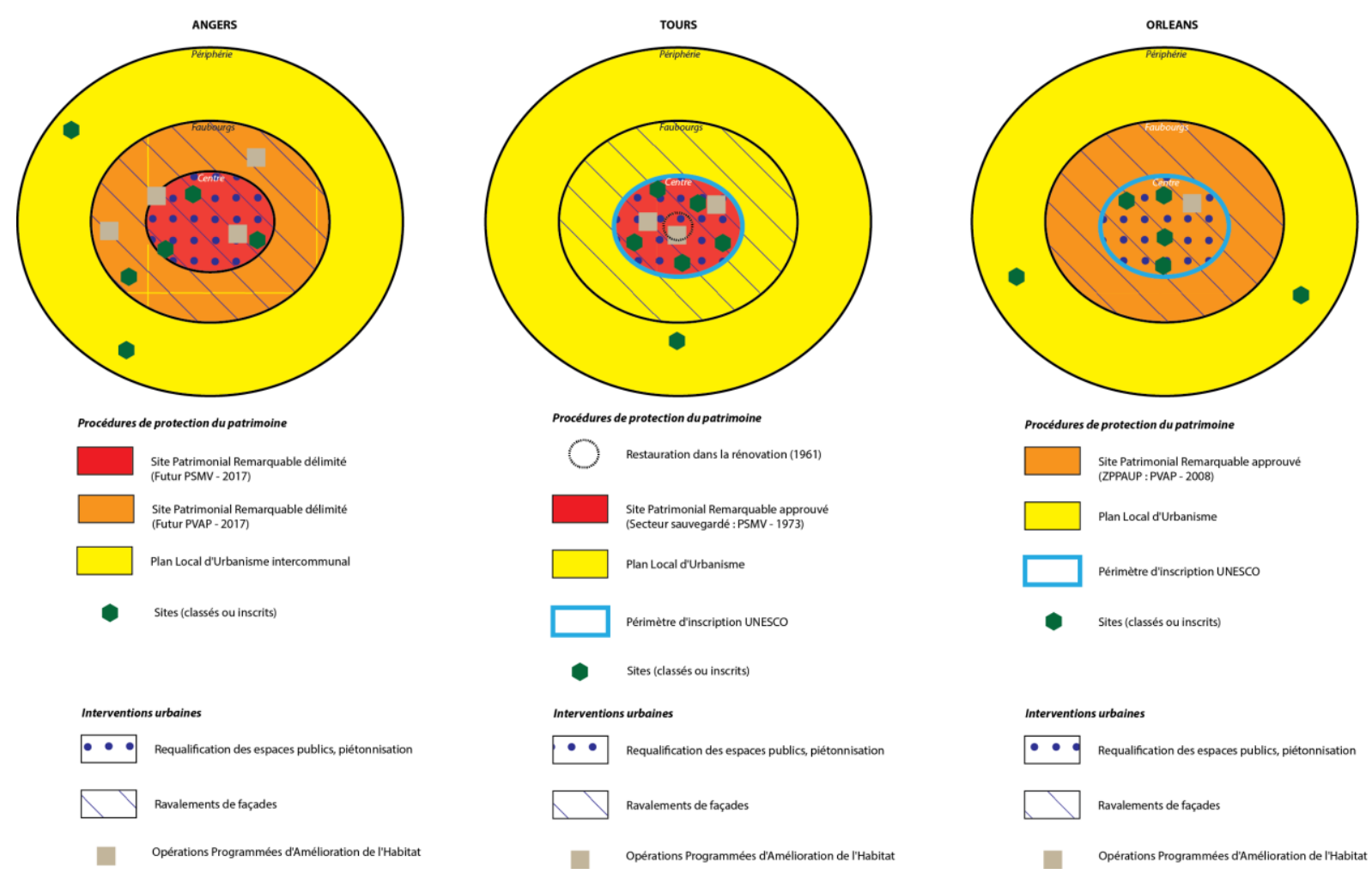

Figure 2 : Schémas des politiques patrimoniales à Angers, Tours et Orléans / Pattern of heritage policies in Angers, Tours and Orléans 